\title{
Interfacial adhesion of nanoparticles in polymer blends by intrinsic fluorescence spectra
}

\author{
J. Yang ${ }^{1}$, G. S. He ${ }^{1}$, B. Zhang ${ }^{2}$, W. A. Luo ${ }^{2}$, X. D. Chen ${ }^{1}$, R. W. Fu' ${ }^{2}$ M. Q. Zhang ${ }^{2 *}$ \\ ${ }^{1}$ Institute of Polymer Science, DSAPM Lab, OFCM Institute, School of Chemistry and Chemical Engineering, \\ Sun Yat-Sen University, Guangzhou 510275, P. R. China \\ ${ }^{2}$ Key Laboratory for Polymeric Composite and Functional Materials of Ministry of Education, School of Chemistry and \\ Chemical Engineering, Sun Yat-sen University, Guangzhou 510275, P. R. China
}

Received 26 December 2010; accepted in revised form 18 March 2011

\begin{abstract}
Intrinsic fluorescence was applied to quantitatively describe the interfacial adhesion of nanoparticles in polystyrene/poly(vinyl methyl ether) (PS/PVME) blends. Due to the aggregation of aromatic rings on PS chains, the temperature dependence of excimer fluorescence intensity $\left(I_{324}\right)$ showed the high sensitivity to the phase separation process. Consistent with Ginzburg thermodynamic model, it was found that the addition of spherical hydrophilic nanoparticles shifted the phase separation temperature to higher temperatures due to the aggregation of silica into PVME chains leading to the free energy reduction and slowing down the phase separation dynamics. A certain composition of polymer blend, i.e. $2 / 8$, was focused on to shed light on the dynamic of spinodal decomposition (SD) phase separation by using decomposition reaction model. It was shown that the addition of nanoparticles to polymer blends resulted in the deviation of linear relationship between the initial SD phase separation rate $\left(R_{\mathrm{p} 0}\right)$ and thermodynamic driving force $\left(\Delta f_{\mathrm{SD}}\right)$. Besides, for PS/PVME $(2 / 8)$ with 2 vol\% silica nanoparticles, the apparent activation energy of phase separation $\left(E_{\mathrm{a}}\right)$ was $196.61 \mathrm{~kJ} / \mathrm{mol}$, which was higher than that of neat PS/PVME $(2 / 8)$ blend $\left(E_{\mathrm{a}}=173.68 \mathrm{~kJ} / \mathrm{mol}\right)$, which strongly confirmed the interfacial adhesion effect of silica nanoparticles as compatibilizers.
\end{abstract}

Keywords: polymer blends and alloys, phase separation, nanoparticles, fluorescence

\section{Introduction}

During the past years, a large number of studies focused on the miscibility and phase separation in polymer blends, from both theoretical and experimental viewpoints [1-6]. For polymer mixtures, controlling components compatibility has always seemed to be a very important factor in polymer industry. Recently, as potential compatibilizers, nanoparticles have attracted more and more attention due to their important role the immiscible polymer blends [7-12]. It has been shown that introducing nanoparticles can make the phase behavior more complicated by changing the shape of the phase diagram [13], changing the interaction param- eter between two components $[14,15]$, and increasing or decreasing the phase separation temperature [13-16].

Among previous studies, a number of analytical methods [17-21] such as thermal analysis, atomic force microscopy (AFM), and scattering methods (visible light, neutron, X-ray) have been frequently used to investigate phase behavior of polymer blends. However, each of these techniques has its advantages and disadvantages, depending on the systems and problems of interest. For instance, the thermal analyses are very convenient to observe the miscibility in polymer blends, but sometimes it is hard to detect $T_{\mathrm{g}} \mathrm{s}$ of polymer blends very clearly.

\footnotetext{
${ }^{*}$ Corresponding author, e-mail: ceszmq@mail.sysu.edu.cn
} (c) BME-PT 
Compared with AFM, light scattering methods are superior because one can analyze the relaxation rate as a function of the scattering vector and that one can follow, in situ, the rapid growth of the fluctuations without introducing an additional physical process such as freezing a given phase-separated state. However, the light scattering technique inevitably depends on the expensive equipment and sophisticated process. As a sensitive method, the fluorescence technology is also widely applied to investigate macromolecular chain movements with probe and labeling [22-24]. Furthermore, it has been proved that the fluorescence technique can provide us with information on a scale smaller than conventional light scattering and comparable to small-angle neutron scattering [25]. Although fluorescence method can obtain lots of useful information of polymer micromorphology and microstructure, the labeling procedure is often tedious. In addition, in most of the fluorimetric studies of polymer, the fluorescent probe was covalently attached to one of the polymers, which in fact changed the microenvironment of macromolecules and made the macromolecules more hydrophobic, thus enhancing their complexation ability. Actually, the intrinsic fluorescence of polymers (e.g. polystyrene) has also been shown to be highly sensitive to issues ranging from local polymer conformational populations in solution and phase behavior in solvents and polymer blends to local microenvironments in bulk homopolymers [26-30].

Thus, intrinsic fluorescence was employed to investigate the phase separation behavior of polymer

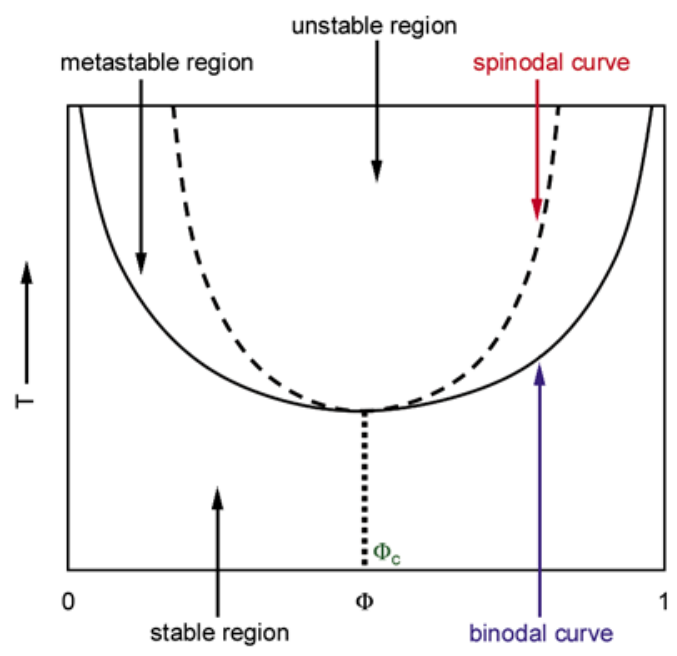

Figure 1. Schematic phase diagram of a polymer blend which exhibits a lower critical solution temperature (LCST) blends in our work. Here, polystyrene/poly(vinyl methyl ether) (PS/PVME) blend has been chosen as a model system. This blend exhibits a typical lower critical solution temperature (LCST) behavior [3133], which is illustrated by the schematic polymerpolymer phase diagram in Figure 1. In this work, the interfacial adhesion of nanoparticles in PS/PVME blends has been quantitatively characterized by intrinsic fluorescence combining with a simple model based on decomposition reaction.

\section{Experimental}

\subsection{Materials}

PS $\left(M_{\mathrm{w}}=354000 \mathrm{~g} / \mathrm{mol}, \mathrm{PDI}=1.05\right.$, Polymer Source Inc., Canada $)$ and PVME $\left(M_{\mathrm{w}}=90600 \mathrm{~g} / \mathrm{mol}, \mathrm{PDI}=\right.$ 1.95, Scientific Polymer Products Inc., U. S. A.) were used in this study. Silica nanoparticles (Aerosil 200) were supplied by Evonik-Degussa Co., (Germany) with an average diameter of $12 \mathrm{~nm}$ and a specific surface area of $200 \mathrm{~m}^{2} / \mathrm{g}$. The particles were dried at $120^{\circ} \mathrm{C}$ under vacuum for $24 \mathrm{~h}$ to eliminate the physically absorbed and weakly chemically absorbed species. The PS/PVME/nanoparticles samples were prepared by continuous mechanical mixing of the components in toluene. And then, film samples were prepared by casting from $10 \mathrm{wt} \%$ toluene solutions of the polymer blend containing onto quartz plates at room temperature. The films were further heat treated in a vacuum oven at $45^{\circ} \mathrm{C}$ for $4 \mathrm{~h}$ and then $65^{\circ} \mathrm{C}$ for $24 \mathrm{~h}$ in order to remove the residual solvent. The vacuum was applied slowly to prevent any possible bubble formation. We found this procedure resulted in homogeneous films which were slightly opaque. The thickness of film was about $3 \mu \mathrm{m}$.

\subsection{Methods}

All the fluorescence emission spectra were recorded using a FLS920 Combined Fluorescence Lifetime and Steady State Spectrometer (Edinburgh, England) in front-face geometry. The excitation wavelength is at $260 \mathrm{~nm}$ and the slit (ex/em) width of the measurements was $1.5 \mathrm{~nm} / 2.5 \mathrm{~nm}$. The sample is heated by conduction via a nitrogen exchange gas in the sample space and the temperature control is achieved by a combination of manual nitrogen flow control and power dissipated in an electrical heater, regulated using an ITC temperature controller. By using this temperature-controlling accessory, the 
variable-temperature measurements within $77-500 \mathrm{~K}$ can be carried out with the stability of $\pm 0.1 \mathrm{~K}$. In non-isothermal measurement, the sample was heated at a rate of $0.5 \mathrm{~K} / \mathrm{min}$ with a time interval of $10 \mathrm{~s}$ for each increase of $2.5 \mathrm{~K}$, while in isothermal measurement, the sample was heated to a specified temperature within 1 minute and then kept for $15 \mathrm{sec}-$ onds.

\section{Results and discussion \\ 3.1. Phase separation temperature}

Figure 2 shows the intrinsic fluorescence spectra of PS/PVME (2/8) film at different temperatures. It is clear that, for each spectrum, a maximum intensity can be observed at $324 \mathrm{~nm}$, with a second, lesser peak at $283 \mathrm{~nm}$. It is worth mentioning that, intrinsic fluorescence of PS consisted of both monomer and excimer fluorescence, the former due to emission from a single excited-state phenyl ring, while the latter due to the emission from an excited-state dimer consisting of two phenyl rings in a parallel, sandwich-like conformation with an inter-ring separation distance of 3-4 $\AA$ [34-36]. Hence, it is easy to understand that the intrinsic fluorescence peak at 324 and $283 \mathrm{~nm}$ should be ascribed to the excimer and monomer fluorescence, respectively. For PS, there exist three types of nominal excimer forming sites: (i) intermolecular interaction between rings on different chains (ii) intramolecular interaction between rings on nonadjacent chain segments and (iii) intramolecular site formed between aromatic in Figure 2, the intrinsic fluorescence intensity in the whole wavelength range increases as the temperature increases in the temperature range studied

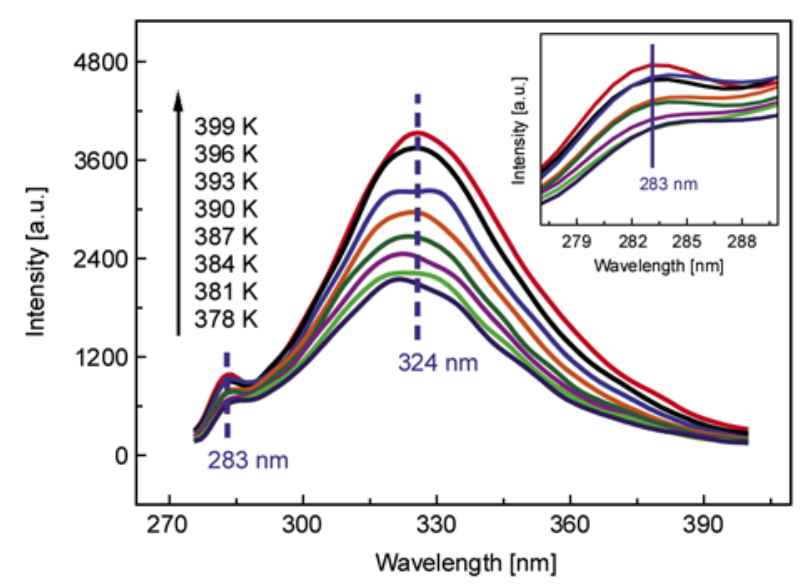

Figure 2. The intrinsic fluorescence spectra of PS/PVME $(2 / 8)$ film at different temperatures. The inset shows enlargement of emission peak at $283 \mathrm{~nm}$ here. It is worth mentioning that the excited state of chromophore promoted by the absorption of a photon is usually deactivated by radiative (fluorescence $\left.K_{\mathrm{r}}\right)$, and non-radiative $\left(K_{\mathrm{nr}}\right)$ and radiative energy transfer rates $\left(K_{\mathrm{RET}}\right)$, thus the change in intrinsic fluorescence intensity $(I)$ can be understood considering Equation (1) for the fluorescence quantum yield $\left(\phi_{\mathrm{F}}\right)$ :

$I \propto \phi_{\mathrm{F}}=\frac{K_{\mathrm{r}}}{K_{\mathrm{r}}+K_{\mathrm{nr}}+K_{\mathrm{RET}}}$

Since $K_{\mathrm{r}}$ only depends on temperature through the refraction index [40], Equation (1) can be expressed as Equation (2):

$I \propto \frac{K_{\mathrm{r}}^{0} n^{2}}{K_{\mathrm{r}}^{0} n^{2}+K_{\mathrm{nr}}+K_{\mathrm{RET}}}$

where $K_{\mathrm{r}}^{0}$ is a constant independent of temperature and $n$ is the refraction index. Therefore, from Equation (2), it can be seen that intrinsic fluorescence is mainly influenced by $n, K_{\mathrm{nr}}$ and $K_{\mathrm{RET}}$. Based on this point, the changes in intrinsic fluorescence intensity of excimer and monomer should be interpreted in terms of the balance of these three factors: (i) the reduction of the refraction index [41]; (ii) the deactivation of the fluorescence by non-radiative processes due to the increased thermal motions, and (iii) the attenuated radiative energy transfer caused by the decreased the local concentration of chromophore during volume expansion [42]. The detailed mechanism will be mentioned in the next section. In general, the results shown in Figure 2 reveal the sensitivity of the intrinsic fluorescence in the phase separation process of PS/PVME film.

Since the minor emission (monomer) was gradually affected by the adjacent major emission (excimer) in the fluorescence spectra during phase separation so that its intensity change could not reflect the actual conformational transitions. Thus, we only focused our attention on fluorescence intensity of excimer $\left(I_{324}\right)$ rather than monomer $\left(I_{283}\right)$. As shown in Figure 3, $I_{324}$ first decreases slightly with the increasing temperature and then sharply increases at $368 \mathrm{~K}$. It is noteworthy that the fluorescence intensity, which is proportional to the fluorescence quantum yield, is expected to decrease with increasing temperature because of an enhancement of nonradiative decay rates with increasing thermal energy. Besides, the change in the temperature dependence 
at $T_{\mathrm{g}}$ is a manifestation of the change in the temperature dependence of polymer density which also affects non-radiative decay processes [43]. It can be seen that a faster decrease in the intrinsic fluorescence intensity appears at $T>T_{\mathrm{g}}$ of PS, although the degree to which the intensity declines is small [43]. Thus, the sharp rise in fluorescence intensity should be taken as the signature of phase separation of PS/PVME. For comprehensive understanding of this behavior, the temperature dependence of excimer to monomer fluorescence intensity ratio $\left(I_{324} / I_{283}\right)$ is also taken into account since excitedstate monomer and excimer can be interconvertible during aggregation of PS [37-39]. Obviously, the value of $I_{324} / I_{283}$ does not change so much and starts to enhance at above $368 \mathrm{~K}$ (see Figure 3). As we know, $I_{324} / I_{283}$ depends upon two major factors [37]: (i) the electronic stability of the excimer complex, as manifested through the radiative and nonradiative fluorescence decay constants of the excimer and monomer which usually are not expected to vary appreciably and (ii) the number of excimerforming sites [44] including the intramolecular site formed between aromatic rings on adjacent and/or nonadjacent repeat units on the same chain, and the intermolecular site formed between aromatic rings on different polymer chains. Thus, the explanation of variation of $I_{324}$ and $I_{324} / I_{283}$ should now be clear. At $T<368 \mathrm{~K}$, the invariant value of $I_{324} / I_{283}$ suggests that almost no conversion between monomer and excimer occurs, and thus the reduction of $I_{324}$ should be attributed to the decrease of excimer fluorescence quantum yield caused by the enhance-

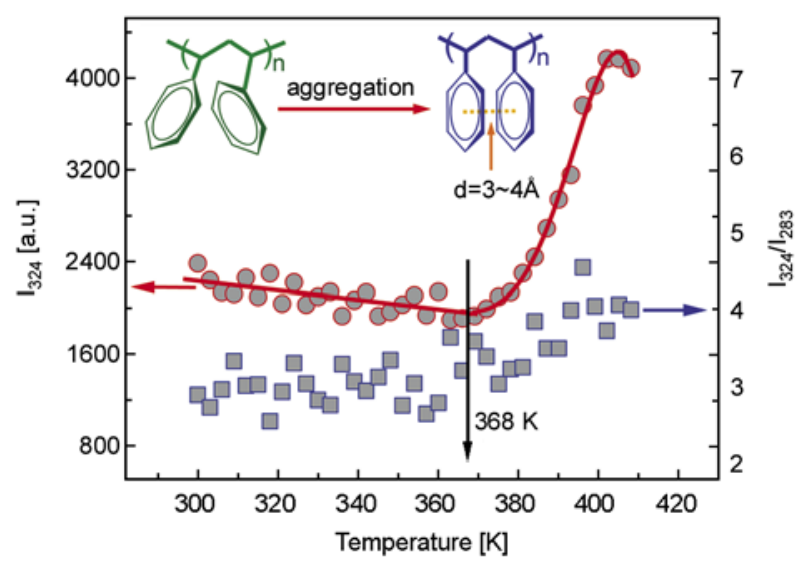

Figure 3. Temperature dependence of excimer fluorescence intensity $\left(I_{324}\right)$ and excimer to monomer fluorescence intensity ratio $\left(I_{324} / I_{283}\right)$ for the PS/PVME (2/8) film. Inset shows the conformational change of PS during aggregation process ment of non-radiative decay rate [24] before phase separation. At $T>368 \mathrm{~K}$, the aggregation of PS chains during phase separation results in the increase of the number of both intramolecular and intermolecular excimer sites, i.e. an increased probability of remote segment contact on the same chain and clustering of segments on different chains (see inset of Figure 3), leading to the increase of $I_{324}$ and $I_{324} / I_{283}$. Due to the more distinguishable change of excimer fluorescence intensity during aggregation, only $I_{324}$ will be considered for investigating the phase separation behavior in the following discussion.

In the case of nanoparticle-filled samples, temperature dependence of $I_{324}$ is shown in Figure 4 for the PS/PVME (2/8) film with 2 vol\% silica nanoparticles. In comparison with Figure 3, the intrinsic fluorescence spectra for PS/PVME with and without nanoparticles are very similar (see inset of Figure 4), which suggests fluorescence behavior of chromophores on PS chains in blends are not affected by the addition of nanoparticles. However, the inflection point for PS/PVME (2/8) film with $2 \%$ silica nanoparticles shifts to a higher temperature ( $375 \mathrm{~K}$ ). This phenomenon should be attributed to the change of network structure in the blend caused by interfacial adhesion. Note that PVME molecules (dispersive solubility parameter, $\delta_{\mathrm{d}}=15.5 \mathrm{MPa}^{1 / 2}$, and polar solubility parameter, $\delta_{\mathrm{p}}=7.1 \mathrm{MPa}^{1 / 2}$ ) are much more polar than PS molecules $\left(\delta_{\mathrm{d}}=\right.$ 18.1 $\mathrm{MPa}^{1 / 2}$ and $\delta_{\mathrm{p}}=1.1 \mathrm{MPa}^{1 / 2}$ ) [45] so that PVME chains tended to be preferentially absorbed on the nanoparticles surface. On the other hand, due to great thermodynamic work of adhesion between PS

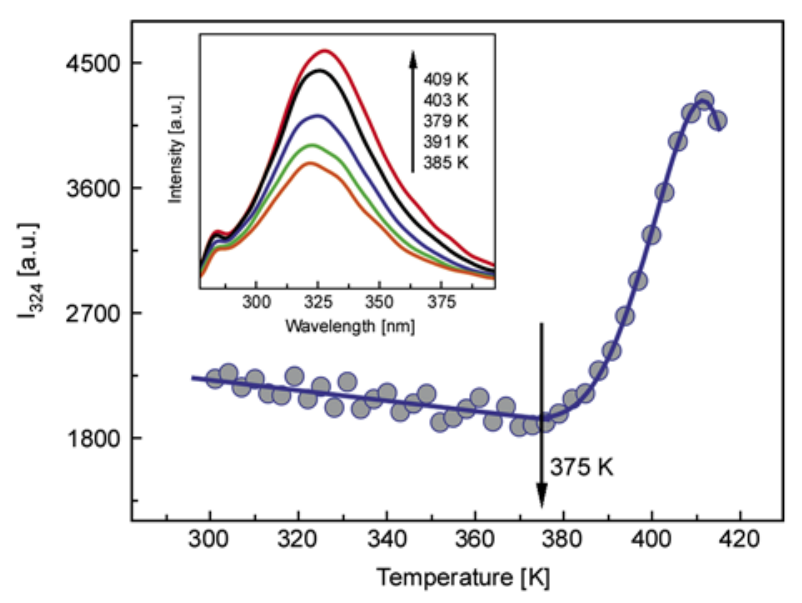

Figure 4. Temperature dependence of $I_{324}$ for PS/PVME (2/8) film with 2 vol\% silica nanoparticles. The inset shows the intrinsic fluorescence spectra at different temperatures 
and PVME [46], the PS/PVME interface is still relatively stable under the shear forces during mixing so that nanoparticles more likely exist in PVME but not at the interphase between PS and PVME, forming the final core-shell structure which has been confirmed in scanning electron microscopy results obtained by Gharachorlou and Goharpey [46]. When the SD phase separation occurs, the low mobility region absorbed on nanoparticles would act like a network of obstacles for the coarsening of the interfaces and slow down the transition from interpenetrated structure to matrix-disperse [47]. Thus, the interconnected network can remain in its initial state for a longer time, showing a higher phase separation temperature which will be discussed in the following section.

Figure 5 shows the phase separation temperature curve determined by intrinsic fluorescence for PS/ PVME blends with and without $2 \%$ silica nanoparticles. For each case, the extremum temperature appears at the low PS volume fraction (i.e. $\Phi_{\mathrm{c}}=0.2$ ), resulting from the higher molecular weight of one component (i.e. PS) [48]. Besides, as we know, the phase separation of PS/PVME film usually undergoes two different mechanisms, i.e. spinodal decomposition (SD) and nucleation and growth (NG) [33]. This critical PS volume fraction strongly suggests the phase separation of PS/PVME blend (2/8) that we focused on occurs only by SD mechanism since the binodal curve and spinodal curve intersect at $\Phi_{\mathrm{c}}$ (shown in Figure 1). Recently, Ginzburg [16] pro-

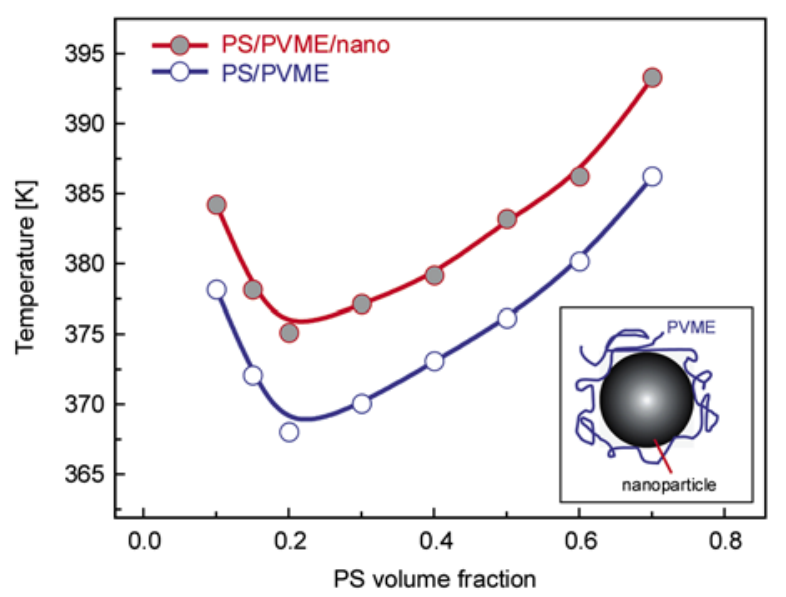

Figure 5. Phase separation temperature curve determined by intrinsic fluorescence for the PS/PVME blends with and without $2 \%$ silica nanoparticles The inset shows the schematic representation for spherical nanoparticles covered with PVME chains. posed a simple model describing the thermodynamics of two homopolymers (A and B) with spherical nanoparticles (covered with an A-polymer layer). Here, the A-polymer refers to PVME (see inset of Figure 5). From his theory, one can obtain the effective decrease in the spinodal temperature of an upper critical solution temperature (UCST)-type binary blend in the presence of nanoparticles (Equation (3)):

$\frac{\Delta T_{s p}}{T_{\mathrm{sp}}} \approx-\psi$

where $\psi$ and $T_{\mathrm{sp}}$ are the nanoparticle volume fraction and spinodal temperature of the neat binary blend, respectively. From Equation (3), it follows that for a binary blend $(\Phi=0.2)$ with a critical temperature of $368 \mathrm{~K}$, addition of $2 \mathrm{vol} \%$ nanoparticles can reduce the spinodal temperature by about $7 \mathrm{~K}$. Naturally, for LCST-type blends, the situation would be the opposite. In our work, addition of 2 vol\% fumed silica caused the spinodal temperature to shift from 368 to $375 \mathrm{~K}$ for PS/PVME (2/8) blends. Therefore, the model proposed by Ginzburg successfully predicts the interfacial adhesion of nanoparticles in PS/PVME system.

\subsection{Kinetics of whole process of phase separation.}

To further characterize the interfacial adhesion of silica nanoparticles, the isothermal phase separation was also studied. The intrinsic fluorescence spectra of PS/PVME (2/8) film at $370 \mathrm{~K}$ at different times are presented in Figure 6. As expected, the time dependence of $I_{324}$ shows that the intensity of spectra first steeply increases with the temperature increased, and then levels off which indicated the completion of phase separation process (see inset of Figure 6). The similar behavior has also been found in the case of PS/PVME with silica nanoparticles. Hereafter, $I_{324}$ is used to perform a quantitative analysis of the kinetics for PS/PVME with and without nanoparticles.

Taking into account the slight fluorescence difference induced by the different thickness of films, the relative extent of phase separation $(\alpha)$ at time thas been considered. $\alpha$ is defined as Equation (4):

$\alpha=\frac{I_{\mathrm{t}}-I_{0}}{I_{\infty}-I_{0}}$ 


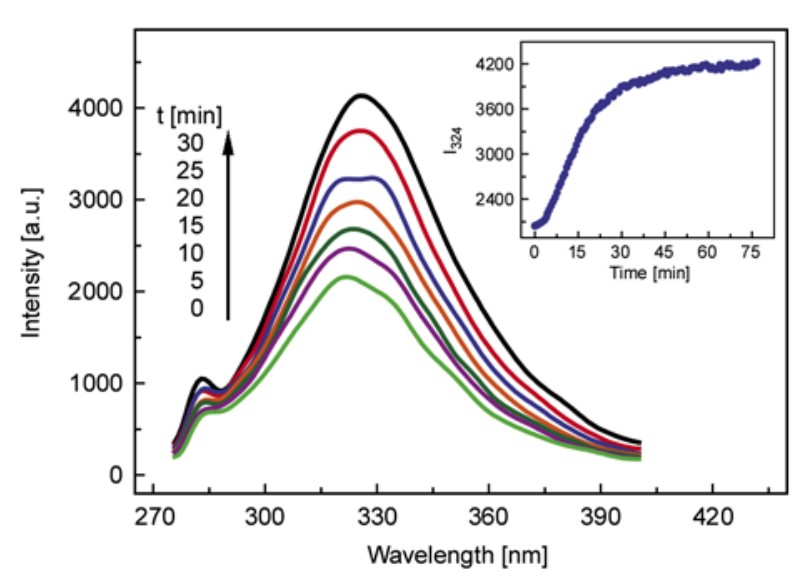

Figure 6. The intrinsic fluorescence spectra of PS/PVME (2/8) film during the isothermal phase separation at $370 \mathrm{~K}$. The inset shows the time dependence of $I_{324}$

where $I_{\mathrm{t}}, I_{0}$ and $I_{\infty}$ are $I_{324}$ for phase separation at time $t, 0$ and $\infty$, respectively. Time dependences of relative extent of phase separation of PS/PVME (2/8) film are shown in Figure 7. It can be seen that, the higher the temperature was, the faster the phase separation occurred, which was characteristic phase separation behavior of LCST polymer blend. The curves for PS/PVME/nanoparticles are similar with Figure 6, which are not shown here.

In order to simplify the quantitative calculation of the phase separation kinetics, we proposed a simple model based on decomposition reaction (see Figure 8), assuming nanoparticles always remains in the PVME phase. When the phase separation takes place, the 'reactant' (A), i.e. PS/PVME or PS/PVME/ nanoparticles, would be decomposed into two products, i.e., (B) PS and (C) PVME or PVME/nanoparticles. As far as the 'decomposition reaction' with one 'reactant' is concerned, the evidence in favor of

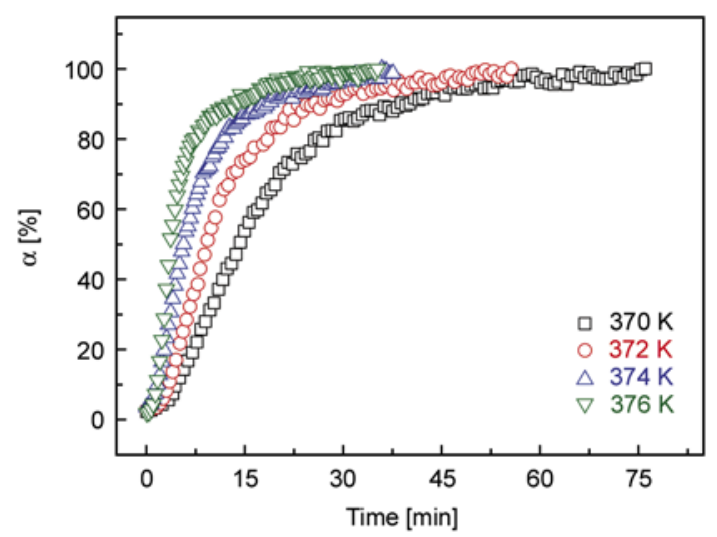

Figure 7. Time dependences of relative extent $(\alpha)$ of phase separation of PS/PVME (2/8) film at various temperatures

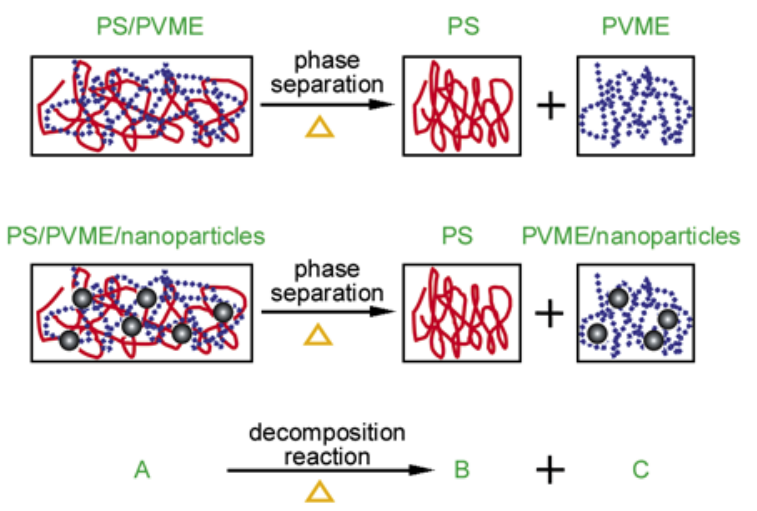

Figure 8. Schematic illustration of phase separation of PS/PVME/nanoparticles

the first order reaction is quite convincing, which means the rate of reaction is directly proportional to the concentration of one of the reactants $[49,50]$. As a result, the rate of $\mathrm{SD}\left(R_{\mathrm{p}}\right)$ can be simply expressed by Equation (5) [51]:

$R_{\mathrm{p}}=-\frac{\mathrm{d}[A]}{\mathrm{d} t}=k_{\mathrm{p}}[A]$

where $[A]$ stands for the weight ratio of un-phaseseparated polymer blends to entire polymer blends (i.e. phase-separated part + un-phase-separated part), and $k_{\mathrm{p}}$ is the rate constant of SD. The integrated form of Equation (5) is shown in Equation (6):

$\ln \frac{[A]_{0}}{[A]_{\mathrm{t}}}=k_{\mathrm{p}} t$

where $[A]_{0}$ and $[A]_{\mathrm{t}}$ are the values of $[A]$ at time 0 and $t$, respectively. On this basis, the initial SD phase separation rate $\left(R_{\mathrm{p} 0}\right)$ can be obtained by inputting the values of $k_{\mathrm{p}}$ and $[A]_{0}$ into Equation (5). As expected [52], a linear relationship $(|R|=$ 0.9958 ) is found for neat PS/PVME blend between $R_{\mathrm{p} 0}$ and thermodynamic driving force $\left(\Delta f_{\mathrm{SD}}\right)$, i.e. (see Figure 9). Interestingly, however, in the case of PS/PVME blend with nanoparticles, with the best fit $(|R|=0.9998), R_{\mathrm{p} 0}$ does not increases linearly not quadratically with $\Delta f_{\mathrm{SD}}$, which suggests a stronger dependence of phase separation rate on the thermodynamic factor in addition of nanoparticles. Since the interfacial tension changes with the temperature changes [53], this behavior is probably due to the temperature dependence of preferential surface adsorption of silica nanoparticles in the polymer blend at the higher temperature.

On the other hand, since the relative extent of phase separation $(\alpha)$ can be understood as the percent con- 


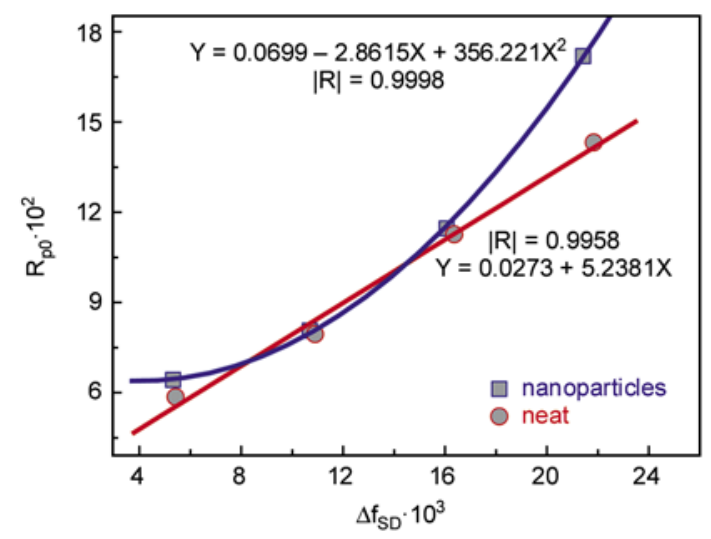

Figure 9. The dependence of SD initial rates on the thermodynamic driving force for the PS/PVME blends with and without $2 \%$ silica nanoparticles

version of decomposition reaction at time $t$, the Equation (6) can be written as shown in Equation (7):

$\ln \frac{[A]_{0}}{[A]_{\mathrm{t}}}=\ln \frac{1}{1-\alpha}$

Therefore, the relationship between $\alpha$ and $R_{\mathrm{p}}$ can be expressed by Equation (8):

$\ln (1-\alpha)=-k_{\mathrm{p}} t$

Figure 10 exhibits plots of $\ln (1-\alpha) v s$. time of phase separation for PS/PVME (2/8) film. By using Equation (8), $k_{\mathrm{p}}$ can be easily obtained from the slopes of linear-fit lines in Figure 10 (see Table 1). Similarly, the phase separation rate constants for PS/PVME/nanoparticles were also calculated in this way. As can be seen in Table 1, all the absolute values of correlation coefficients $(R)$ are in the range of $0.97-0.99$ (very close to 1 ), which strongly

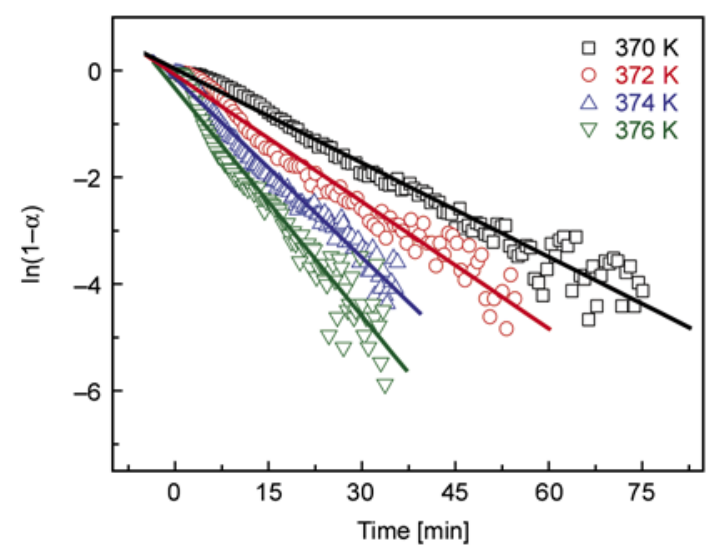

Figure 10. Plots of $\ln (1-\alpha)$ against time of phase separation for PS/PVME (2/8) film
Table 1. Phase separation kinetics parameters of PS/PVME $(2 / 8)$ film with and without 2 vol\% silica nanoparticles determined by intrinsic fluorescence spectroscopy

\begin{tabular}{|l|c|c|c|c|}
\hline PS/PVME (2/8) & $\mathbf{T}[\mathbf{K}]$ & $\mathbf{k}_{\mathbf{P}}\left[\mathbf{m i n}^{-\mathbf{1}}\right]$ & $|\mathbf{R}|$ & $\mathbf{E}_{\mathbf{g}}[\mathbf{k J} / \mathbf{m o l}]$ \\
\hline \multirow{4}{*}{ neat } & 370 & 0.05888 & 0.98221 & \\
\cline { 2 - 4 } & 372 & 0.07957 & 0.98292 & \multirow{4}{*}{173.68} \\
\cline { 2 - 4 } & 374 & 0.11249 & 0.98825 & \\
\cline { 2 - 4 } & 376 & 0.14281 & 0.97171 & \\
\hline \multirow{4}{*}{ nanoparticles } & 377 & 0.06448 & 0.98326 & \multirow{4}{*}{196.61} \\
\cline { 2 - 4 } & 379 & 0.08075 & 0.98424 & \\
\cline { 2 - 4 } & 381 & 0.11445 & 0.98649 & \\
\cline { 2 - 4 } & 383 & 0.17125 & 0.95751 & \\
\hline
\end{tabular}

confirms the above kinetics analysis based on the decomposition reaction assumption is reasonable.

To better describe the adhesion effect of nanoparticles in the polymer blends, apparent activation energy, $E_{\mathrm{a}}$, of phase separation are obtained by applying Arrhenius equation $\left(k_{\mathrm{p}}=A \exp \left(-E_{\mathrm{a}} / R T\right)\right)$ here. Figure 11 presents the plot of $\ln k_{\mathrm{p}}$ against the reciprocal of temperature for the PS/PVME (2/8) with and without nanoparticles. Obviously, for each case, the rate constant $k_{\mathrm{p}}$ decreases with temperature increases and lnkp $\sim 1 / T$ plot shows a good linear relation. Thus, $E_{\mathrm{a}}$ can be calculated from the slope of lines. As listed in Table 1, the apparent activation energy of the neat PS/PVME blend $\left(E_{\mathrm{a}}=\right.$ $173.68 \mathrm{~kJ} / \mathrm{mol}$ ) was lower than that of the PS/PVME blend with nanoparticles $\left(E_{\mathrm{a}}=196.61 \mathrm{~kJ} / \mathrm{mol}\right)$. These values are very similar to apparent activation energy of phase separation for PS/PVME (2/8), $194.68 \mathrm{~kJ} / \mathrm{mol}$, obtained by resonance light scattering method [55]. It is worthwhile to mention that in the case of SD, the phases are interconnected at the initial stage of phase separation, during which the

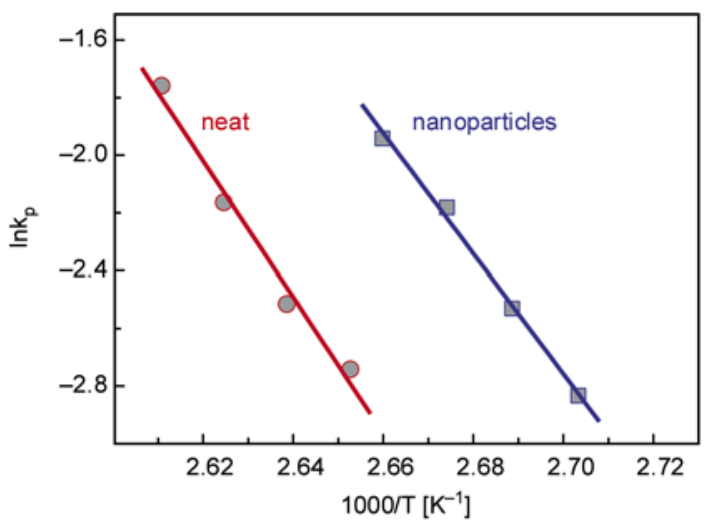

Figure 11. Plot of lnkp film against the reciprocal of temperature for the PS/PVME (2/8) with and without nanoparticles 
domains are constant in size. With the time or temperature increases, the phase domains then grow in size while maintaining their connectivity, while in the latter stages the phases break into small spheres and then merge into macrospheres [54]. Note the phase growth is diffusion controlled, thus this apparent activation energy is a key parameter for characterizing the diffusion rate of macromolecular chains in polymer blends. So one can easily understand that the lower its value is, the easier the cocontinuous network structure can be formed and transformed into macro-separated phases. This result highly consists with the results shown in Figure 5 , clarifying the interfacial adhesion of silica nanoparticles.

\section{Conclusions}

In this work, the interfacial adhesion of nanoparticles in PS/PVME blends was studied by intrinsic fluorescence method. By monitoring the temperature dependence of excimer fluorescence intensity, it can be seen that the addition of spherical hydrophilic nanoparticles shifted the phase separation temperature to higher temperatures. This behavior was due to the absorption of some PVME chains on nanoparticles surface which assisted the free energy reduction and the stability of the homogeneous phase, slowing down the phase separation dynamics. Furthermore, a simple model based on the decomposition reaction was proposed to perform the quantitative analysis of the SD phase separation kinetics. Compared with neat blend, a stronger dependence of phase separation rate on the thermodynamic driving force was observed in the PS/ PVME (2/8) with 2 vol\% silica nanoparticles. The results of apparent activation energy strongly confirmed the interfacial adhesion of silica nanoparticles in PS/PVME blends.

\section{Acknowledgements}

Financial support from the program of National Natural Science Foundation of China (Grant no. 50673104 and 50673101) and Natural Science Foundation of Guangdong province (Grant no. 7003702) are gratefully acknowledged.

\section{References}

[1] Kwei T. K., Nishi T., Roberts R. F.: A study of compatible polymer mixtures. Macromolecules, 7, 667-674 (1974). DOI: $\underline{10.1021 / \mathrm{ma} 60041 \mathrm{a} 023}$
[2] Nishi T., Wang T. T., Kwei T. K.: Thermally induced phase separation behavior of compatible polymer mixtures. Macromolecules, 8, 227-234 (1975).

DOI: $10.1021 / \mathrm{ma60044a025}$

[3] Hashimoto T., Kumaki J., Kawai H.: Time-resolved light scattering studies on kinetics of phase separation and phase dissolution of polymer blends. 1. Kinetics of phase separation of a binary mixture of polystyrene and poly(vinyl methyl ether). Macromolecules, 16, 641-648 (1983).

DOI: $10.1021 / \mathrm{ma} 00238 \mathrm{a} 030$

[4] Snyder H. L., Meakin P., Reich S.: Dynamical aspects of phase separation in polymer blends. Macromolecules, 16, 757-762 (1983).

DOI: $10.1021 / \mathrm{ma} 00239 \mathrm{a} 011$

[5] Hill R. G., Tomlins P. E., Higgins J. S.: Preliminary study of the kinetics of phase separation in high molecular weight poly(methyl methacrylate)/solution-chlorinated polyethylene blends. Macromolecules, 18, 25552560 (1985).

DOI: $10.1021 / \mathrm{ma} 00154 \mathrm{a} 033$

[6] Kumaki J., Hashimoto T.: Time-resolved light scattering studies on kinetics of phase separation and phase dissolution of polymer blends. 4 . Kinetics of phase dissolution of a binary mixture of polystyrene and poly (vinyl methyl ether). Macromolecules, 19, 763-768 (1986).

DOI: $10.1021 / \mathrm{ma} 00157 \mathrm{a} 046$

[7] Ginzburg V. V., Qiu F., Paniconi M., Peng G., Jasnow D., Balazs A. C.: Simulation of hard particles in a phase-separating binary mixture. Physical Review Letters, 82, 4026-4029 (1999).

DOI: $10.1103 /$ PhysRevLett.82.4026

[8] Ginzburg V. V., Peng G., Qiu F., Jasnow D., Balazs A. C.: Kinetic model of phase separation in binary mixtures with hard mobile impurities. Physical Review E, 60, 4352-4359 (1999).

DOI: 10.1103/PhysRevE.60.4352

[9] Balazs A. C., Ginzburg V. V., Qiu F., Peng G., Jasnow D.: Multi-scale model for binary mixtures containing nanoscopic particles. The Journal of Physical Chemistry B, 104, 3411-3422 (2000).

DOI: $10.1021 / j p 993356+$

[10] Zhu Y-J., Ma Y-Q.: Phase separation of a polymer blend driven by oscillating particles. Physical Review E, 67, 041503/1-041503/7 (2003). DOI: 10.1103/PhysRevE.67.041503

[11] Laradji M., MacNevin G.: Phase separation dynamics in binary fluids containing quenched or mobile filler particles. The Journal of Chemical Physics, 119, 22752283 (2003). DOI: $10.1063 / 1.1585011$

[12] Laradji M.: A Langevin dynamics study of mobile filler particles in phase-separating binary systems. The Journal of Chemical Physics, 120, 9330-9334 (2004). DOI: $10.1063 / 1.1704636$ 
[13] Nesterov A. E., Lipatov Y. S., Horichko V. V., Gritsenko O. T.: Filler effects on the compatibility and phase separation kinetics of poly(vinyl acetate)poly(methyl methacrylate) mixtures. Polymer, 33, 619-622 (1992).

DOI: 10.1016/0032-3861(92)90740-N

[14] Nesterov A. E., Lipatov Y. S.: Compatibilizing effect of a filler in binary polymer mixtures. Polymer, 40, 1347-1349 (1999).

DOI: $10.1016 / \mathrm{S} 0032-3861(98) 00277-8$

[15] Lipatov Y. S., Nesterov A. E., Ignatova T. D., Nesterov D. A.: Effect of polymer-filler surface interactions on the phase separation in polymer blends. Polymer, 43, 875-880 (2002).

DOI: $10.1016 / \mathrm{S} 0032-3861(01) 00632-2$

[16] Ginzburg V. V.: Influence of nanoparticles on miscibility of polymer blends. A simple theory. Macromolecules, 38, 2362-2367 (2005). DOI: $10.1021 / \mathrm{ma} 0482821$

[17] El-Mabrouk K., Belaiche M., Bousmina M.: Phase separation in PS/PVME thin and thick films. Journal of Colloid and Interface Science, 306, 354-367 (2007).

DOI: $10.1016 /$ j.jcis.2006.10.051

[18] Wignall G. D., Alamo R. G., Londono J. D., Mandelkern L., Stehling F. C.: Small-angle neutron scattering investigations of liquid-liquid phase separation in heterogeneous linear low-density polyethylene. Macromolecules, 29, 5332-5335 (1996).

DOI: $10.1021 / \mathrm{ma} 960050 \mathrm{~h}$

[19] Jha A., Bhowmick A. K.: Mechanical and dynamic mechanical thermal properties of heat- and oil-resistant thermoplastic elastomeric blends of poly(butylene terephthalate) and acrylate rubber. Journal of Applied Polymer Science, 78, 1001-1008 (2000).

DOI: $10.1002 / 1097-4628(20001031) 78: 5<1001:: A I D-$ APP90>3.0.CO;2-G

[20] Swier S., Van Durme K., Van Mele B.: Modulatedtemperature differential scanning calorimetry study of temperature-induced mixing and demixing in poly (vinylmethylether)/water. Journal of Polymer Science Part B: Polymer Physics, 41, 1824-1836 (2003).

DOI: 10.1002/polb.10512

[21] Cabral J. T., Higgins J. S., McLeish T. C. B., Strausser S., Magonov S. N.: Bulk spinodal decomposition studied by atomic force microscopy and light scattering. Macromolecules, 34, 3748-3756 (2001).

DOI: $10.1021 / \mathrm{ma} 0017743$

[22] Klopffer M-H., Bokobza L., Monnerie L.: Structural side effects in the use of a fluorescent probe for monitoring polymer mobility. Macromolecules, 31, 82918296 (1998).

DOI: $10.1021 / \mathrm{ma} 980717 \mathrm{~V}$

[23] Olmos D., González-Benito J.: Cure process and reaction-induced phase separation in a diepoxy-diamine/ PMMA blend. Monitoring by steady-state fluorescence and FT-IR (near and medium range). Colloid and Polymer Science, 284, 654-667 (2006).

DOI: $10.1007 / \mathrm{s} 00396-005-1434-\mathrm{y}$
[24] Corrales T., Peinado C., Bosch P., Catalina F.: Study of secondary relaxations of poly(ethylene terephthalate) by photoluminescence technique. Polymer, 45, 15451554 (2004).

DOI: 10.1016/j.polymer.2003.12.051

[25] Halary J. L., Ubrich J. M., Monnerie L., Yang H., Stein R. S.: Isotope effects on the phase separation in polystyrene-poly(vinylmethylether) blends. Polymer Communications, 26, 73-76 (1985).

[26] Zhang M. Q.: Intrinsic fluorescence: An effective means to monitor macromolecular chain motions. Express Polymer Letters, 2, 835 (2008). DOI: $10.3144 /$ expresspolymlett.2008.97

[27] Tsai F. J., Torkelson J. M.: Phase separation of oligomeric polystyrene-polybutadiene blends as studied by excimer fluorescence. Macromolecules, 21, 1026-1033 (1988). DOI: $10.1021 / \mathrm{ma} 00182 \mathrm{a} 031$

[28] Clauss B., Salem D. R.: A chain-intrinsic fluorescence study of orientation-strain behavior in uniaxially drawn poly(ethylene terephthalate) film. Macromolecules, 28, 8328-8333 (1995).

DOI: $10.1021 / \mathrm{ma} 00128 \mathrm{a} 049$

[29] Ylitalo D. A., Frank C. W.: The effect of pressure on block copolymer micelle formation: Fluorescence and light scattering studies of poly(styrene-b-ethylene propylene) in heptane. Polymer, 37, 4969-4978 (1996). DOI: 10.1016/0032-3861(96)00368-0

[30] Sanz A., Mendicuti F.: Excimers in dilute solutions of $\mathrm{N}$-vinyl carbazole/styrene copolymers of different molar compositions. Polymer, 43, 6123-6130 (2002). DOI: 10.1016/S0032-3861(02)00584-0

[31] Yang H., Shibayama M., Stein R. S., Shimizu N., Hashimoto T.: Deuteration effects on the miscibility and phase separation kinetics of polymer blends. Macromolecules, 19, 1667-1674 (1986).

DOI: $10.1021 / \mathrm{ma} 00160 \mathrm{a} 032$

[32] Wagler T., Rinaldi P. L., Han C. D., Chun H.: Phase behavior and segmental mobility in binary blends of polystyrene and poly(vinyl methyl ether). Macromolecules, 33, 1778-1789 (2000).

DOI: $10.1021 / \mathrm{ma9909105}$

[33] Shibayama M., Stein R S., Han C. C.: Study of miscibility and critical phenomena of deuterated polystyrene and hydrogenated poly(vinyl methyl ether) by small-angle neutron scattering. Macromolecules, 18, 2179-2187 (1985).

DOI: $10.1021 / \mathrm{ma} 00153 \mathrm{a} 020$

[34] Vala M. T., Haebig J., Rice S. A.: Experimental study of luminescence and excitation trapping in vinyl polymers, paracyclophanes, and related compounds. The Journal of Chemical Physics, 43, 886-897 (1965). DOI: $10.1063 / 1.1696866$

[35] Torkelson J. M., Lipsky S., Tirrell M.: Polystyrene fluorescence: Effects of molecular weight in various solvents. Macromolecules, 14, 1601-1603 (1981). DOI: $10.1021 / \mathrm{ma} 50006 \mathrm{a} 094$ 
[36] Torkelson J. M., Lipsky S., Tirrell M., Tirrell D. A.: Fluorescence and absorbance of polystyrene in dilute and semidilute solutions. Macromolecules, 16, 326330 (1983).

DOI: $10.1021 / \mathrm{ma} 00236 \mathrm{a} 031$

[37] Frank C. W., Harrah L. A.: Excimer formation in vinyl polymers. II. Rigid solutions of poly(2-vinylnaphthalene) and polystyrene. The Journal of Chemical Physics, 61, 1526-1541 (1974).

DOI: $10.1063 / 1.1682097$

[38] Frank C. W.: Excimer formation in vinyl polymers. III. Fluid and rigid solutions of poly(4-vinylbiphenyl). The Journal of Chemical Physics, 61, 2015-2022 (1974).

DOI: $10.1063 / 1.1682204$

[39] Frank C. W.: Observation of relaxation processes near the glass transition by means of excimer fluorescence. Macromolecules, 8, 305-310 (1975).

DOI: $10.1021 / \mathrm{ma} 60045 \mathrm{a} 012$

[40] Birks J. B.: Photophysics of aromatics molecules. Wiley, New York (1970).

[41] Beaucage G., Composto R., Stein R. S.: Ellipsometric study of the glass transition and thermal expansion coefficients of thin polymer films. Journal of Polymer Science Part B: Polymer Physics, 31, 319-326 (1993). DOI: $10.1002 /$ polb.1993.090310310

[42] Turrión S. G., Olmos D., Ekizoglou N., GonzálezBenito J.: Fluorescence response from anthracene labeled polystyrene to study its thermal transitions. Polymer, 46, 4023-4031 (2005).

DOI: $10.1016 /$ j.polymer.2005.03.056

[43] Mundra M. K., Ellison C. J., Behling R. E., Torkelson J. M.: Confinement, composition, and spin-coating effects on the glass transition and stress relaxation of thin films of polystyrene and styrene-containing random copolymers: Sensing by intrinsic fluorescence. Polymer, 47, 7747-7759 (2006).

DOI: $10.1016 /$ j.polymer.2006.08.064

[44] Frank C. W., Gashgari M. A.: Excimer fluorescence as a molecular probe of polymer blend compatibility. 1 . Blends of poly(2-vinylnaphthalene) with poly(alkyl methacrylates). Macromolecules, 12, 163-165 (1979). DOI: $10.1021 / \mathrm{ma} 60067 \mathrm{a} 038$

[45] Yurekli K., Karim A., Amis E. J., Krishnamoorti R.: Phase behavior of PS-PVME nanocomposites. Macromolecules, 37, 507-515 (2004).

DOI: $10.1021 / \mathrm{ma} 0302098$
[46] Gharachorlou A., Goharpey F.: Rheologically determined phase behavior of LCST blends in the presence of spherical nanoparticles. Macromolecules, 41, 32763283 (2008).

DOI: $10.1021 / \mathrm{ma} 7020985$

[47] Ginzburg V. V., Qiu F., Paniconi M., Peng G., Jasnow D., Balazs A. C.: Simulation of Hard particles in a phase-separating binary mixture. Physical Review Letters, 82, 4026-4029 (1999).

DOI: $10.1103 /$ PhysRevLett.82.4026

[48] McMaster L. P.: Aspects of polymer-polymer thermodynamics. Macromolecules, 6, 760-773 (1973). DOI: $10.1021 / \mathrm{ma} 60035 \mathrm{a} 024$

[49] Shlyapnikov Y. A., Bogaevskaya T. A., Kiryushkin S. G., Monakhova T. V.: Specific features of formation and properties of hydroperoxides of polyolefins. European Polymer Journal, 15, 737-742 (1979). DOI: 10.1016/0014-3057(79)90024-7

[50] Zlatkevich L.: A chemiluminescence technique for the evaluation of the thermal oxidative stability of polyethylene. Polymer Degradation and Stability, 19, 5160 (1987). DOI: 10.1016/0141-3910(87)90012-7

[51] Benson S. W.: The foundations of chemical kinetics. McGraw-Hill, New York (1960).

[52] Larbi F. B. C., Halary J. L., Monnerie L.: Kinetics of phase separation in binary mixtures of polystyrene and poly(vinyl methyl ether) as studied by fluorescence emission of labeled polystyrene. Macromolecules, 24, 867-871 (1991).

DOI: $10.1021 / \mathrm{ma} 00004 \mathrm{a} 009$

[53] El Mabrouk K., Bousmina M.: Assessment of morphology in transient and steady state regimes resulting from phase separation in polystyrene/poly(vinyl methyl ether) blend. Rheologica Acta, 45, 877-889 (2006). DOI: $10.1007 / \mathrm{s} 00397-005-0076-9$

[54] Langer J. S., Bar-on M., Miller H. D.: New computational method in the theory of spinodal decomposition. Physical Review A, 11, 1417-1429 (1975). DOI: 10.1103/PhysRevA.11.1417

[55] Yang J., Chen X., Fu R., Luo W-L., Li Y., Zhang M.: Kinetics of phase separation in polymer blends revealed by resonance light scattering spectroscopy. Physical Chemistry Chemical Physics, 12, 2238-2245 (2010). DOI: $10.1039 / \mathrm{b} 918069 \mathrm{a}$ 